\title{
High-Speed Quadratic Electrooptic Nonlinearity in dc-Biased InP
}

\author{
L. SubaČIUS ${ }^{a, *}$, I. KaŠAlynaS ${ }^{a}$, M. Vingelis ${ }^{a}$, \\ R. AleKsiejūnas ${ }^{b}$ AND K. JARAŠIUNAAS ${ }^{b}$ \\ ${ }^{a}$ Semiconductor Physics Institute, Goštauto 11, 01108 Vilnius, Lithuania \\ ${ }^{b}$ Institute of Materials Science and Applied Research, Vilnius University \\ Saulétekio 9-3, 10222 Vilnius, Lithuania
}

\begin{abstract}
We present experimental data on degenerate four-wave mixing as well as simulation results of fast optical nonlinearities in highly-excited semiinsulating InP under applied dc-field. Hot-electron transport governed enhancement of optical nonlinearity is obtained by applying a dc-field of $10-14 \mathrm{kV} / \mathrm{cm}$ at full-modulation depth of a light-interference pattern. The hydrodynamic model, which incorporates both free-carrier and photorefractive nonlinearities is used to explain the experimentally observed features. We show that the enhancement of optical nonlinearity is due to the quadratic electrooptic effect.
\end{abstract}

PACS numbers: 71.55.Eq, 72.20.Ht

\section{Introduction}

Photorefractive semi-insulating III-V compounds, such as GaAs and InP that exhibit a negative differential resistance (NDR) and fast optical response, have attracted much interest in recent years because of their possible application in ultrafast semiconductor devices for photonics and optoelectronics. We have shown previously that application of a strong electric field in a region of NDR may lead to very fast and efficient electrooptic (EO) nonlinearity due to nonuniform carrier heating and the photorefractive Gunn-effect. Subnanosecond dynamics of this novel nonlinear effect, observed experimentally in the time-integrated regime under microwave field irradiation of GaAs and $\operatorname{InP}[1,2]$, can be investigated in a dc-biased semiconductor by using a time-resolved picosecond four-wave

*corresponding author; e-mail: liudas@pfi.lt 
mixing (FWM) technique. This technique has demonstrated recently a possibility to generate the photorefractive space-charge $(\mathrm{SC})$ wave of $\mathrm{GHz}$ frequency in nonuniformly photoexcited dc-biased GaAs [3].

In this paper we extend the studies of hot carrier dynamics and transient nonlinear response in dc-biased semi-insulating InP crystal under nonuniform excitation by ps laser beams. The physical processes involved are nonequilibrium carrier dynamics and high-field domain formation in the region of NDR. We demonstrate experimentally that picosecond FWM in highly-excited dc-biased InP exhibits a large enhancement of the EO nonlinearity. Refractive index modulation by coexisting free-carrier (FC) nonlinearity (both electron and hole) and the quadratic EO effect are analyzed numerically by using the hydrodynamic nonlinear transport approach, which enabled us to explain the observed nonlinear features. The numerical studies are performed for conventional parameters of iron-doped semi-insulating InP:Fe when nonequilibrium charge carriers can be generated from the $\mathrm{Fe}^{2+} / \mathrm{Fe}^{3+}$ level and $\mathrm{Fe}^{2+}$ excited state (level $\mathrm{Fe}^{2+*}$ ).

\section{Experimental technique and model}

Nonequilibrium carrier dynamics and nonlinear optical response of the photoexcited InP were studied by monitoring diffraction efficiency of degenerate FWM at various excitation intensities and applied dc electric field strength $E_{\mathrm{dc}}$. The crystal was illuminated by two 30 ps duration coherent laser beams, providing the spatially modulated intensity pattern, $I(x)=I_{0}[1+m \cos (2 \pi x / \Lambda)]$, with the period $\Lambda$ and the modulation index $m=1$. The gratings were recorded in a bulk of the sample and its transient nonlinear response was measured by varying a delay time $\Delta t$ of the probe-beam up to $1 \mathrm{~ns}$. For the experiment, we used a standard semi-insulating InP crystal with a deep iron impurity, responsible for photoconductivity at the used excitation wavelength $\lambda=1.06 \mu \mathrm{m}$ and providing the photogenerated carrier density up to $10^{16} \mathrm{~cm}^{-3}$. The external electric field was orientated along the grating vector.

For numerical studies, a hydrodynamic approach [1] based on hot-electron velocity and mean energy rate equations was used together with a conventional set of the material equations for photoexcited InP:Fe [4]. We calculated spatial profiles of carrier and internal electric field distribution as well as their ps dynamics in subnanosecond time-domain. The amplitudes and phases of FC and EO nonlinearities have been calculated, and their contributions to light diffraction were used to explain the experimentally observed features. We note that, in this work for the used (001)-cut InP crystal, the only contributions to the refractive index modulation by FC nonlinearities, $\Delta n_{\mathrm{FC}}=C_{\mathrm{FC}}\left(N / m^{*}+P / m_{p}\right)_{1}$, and by the quadratic-EO effect, $\Delta n_{\mathrm{EO}}=C_{\mathrm{EO}}\left(E_{\mathrm{int}}^{2}\right)_{1}$, were taken into account. Here $N, m^{*}$, and $P, m_{p}$ are the densities and effective masses of electrons and holes, $E_{\text {int }}$ is the internal electric field, and $C_{\mathrm{FC}}, C_{\mathrm{EO}}$ are the modulation coefficients. The subscript "1" denotes the first Fourier components, which governs the Bragg diffraction. 


\section{Results and discussion}

In the absence of the external electric field, the nonequilibrium carrier spatial distribution and their temporal evolution are fully responsible for the refractive index modulation and dynamics of diffraction efficiency in semi-insulating InP under ps laser excitation. The measured kinetics of nonlinear response revealed a very fast component of initial grating decay and the slower one, which dominates later. This is demonstrated in Fig. 1.

The plotted data of nonlinear optical measurements were found in rather good agreement with the theoretical curves, based on the calculated dynamics of photoexcited carrier density, shown in Fig. 1b. Note, in the used InP:Fe model,
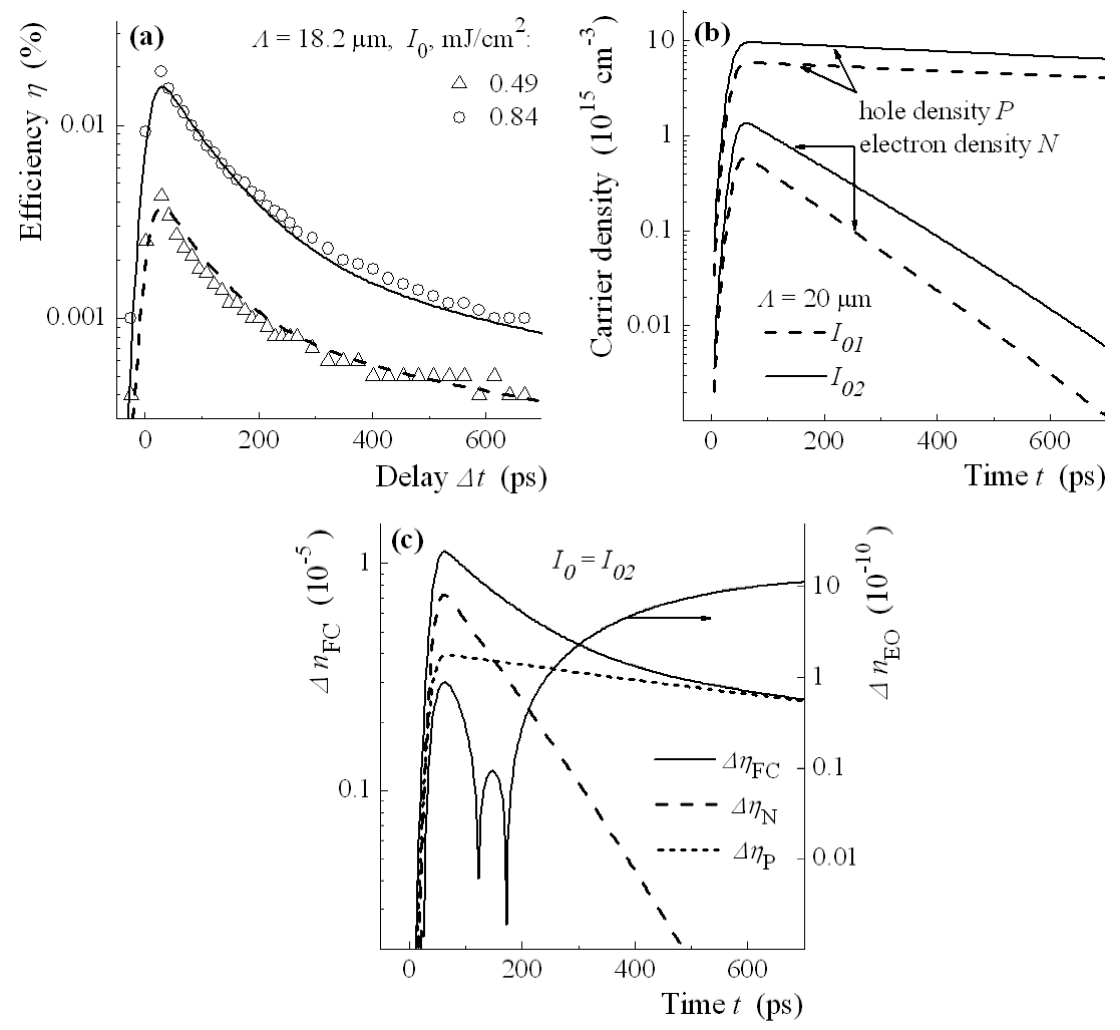

Fig. 1. (a) Kinetics of diffraction efficiency at $E_{\mathrm{dc}}=0$ for grating period of $\Lambda=18.2 \mu \mathrm{m}$ at two different excitation intensities, and (b) simulated carrier density dynamics at illumination by light-interference pattern with $\Lambda=20 \mu \mathrm{m}$ and intensity $I_{0}$, arb.u.: $I_{01}$ (dash curves) and $I_{02}=1.7 \times I_{01}$ (solid curves). (c) Dynamics of refraction index modulation by electrons $\left(\Delta n_{N}\right)$, holes $\left(\Delta n_{P}\right)$, and electron-hole plasma $\left(\Delta n_{\mathrm{FC}}\right)$, calculated using modulation coefficient $C_{\mathrm{FC}}=-3.5 \times 10^{-22} \mathrm{~cm}^{3}$. The right-hand scale stands for the quadratic EO nonlinearity $\Delta n_{\mathrm{EO}}$, calculated for the value of $C_{\mathrm{EO}}=-3 \times 10^{-8} \mathrm{~cm}^{2} / \mathrm{kV}^{2}$. 
the dominant carriers are holes (see Fig. 1b). Since FC nonlinearity is inversely proportional to effective mass and electron-hole concentration varies with time, the combined contribution of electron and hole gratings to refractive index modulation also varies in time: initially the electrons contribute more significantly than the holes, while the hole grating becomes dominant at later time. These peculiarities allow us to explain the measured nonexponential kinetics of grating decay. In Fig. 1c we demonstrate time dependence of the combined refractive index modulation by the FC grating $\left(\Delta n_{\mathrm{FC}}\right)$ and contributions of electron and hole gratings $\left(\Delta n_{N}\right.$ and $\left.\Delta n_{P}\right)$, calculated for the case $I_{0}=I_{02}$ and effective masses $m_{N}^{*} / m=0.078$ and $m_{P}^{*} / m=0.8$. For comparison (see right-hand scale, Fig. 1c) we show the EO refractive index modulation $\Delta n_{\mathrm{EO}}$ (quadratic EO mechanism), calculated for the value of EO modulation coefficient of $C_{\mathrm{EO}}=-3 \times 10^{-8} \mathrm{~cm}^{2} / \mathrm{kV}^{2}$. The latter value, estimated from the GaAs measurements [3], is comparable with $-1.3 \times 10^{-8}$ value, given in Ref. [5] for InP:Fe at $\lambda=1.064 \mu \mathrm{m}$.
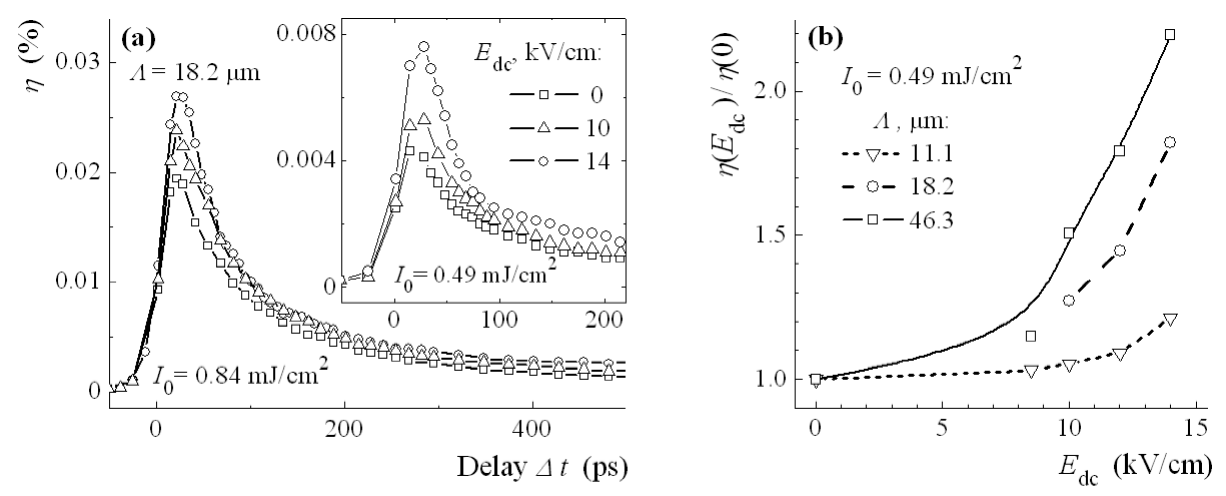

Fig. 2. Experimental results of diffraction efficiency vs. (a) probe-beam delay time and (b) applied dc-field strength in InP:Fe for various external fields, excitation intensity $I_{0}$, and grating period $\Lambda$ values.

Nonlinear transport that governed enhancement of diffraction efficiency was observed in our samples under the applied high dc-field (at $E_{\mathrm{dc}}>10 \mathrm{kV} / \mathrm{cm}$, before illumination). The origin of dc-field induced nonlinearity was attributed mainly to the enhancement of EO nonlinearity due to the high-field domain formation in the region of NDR. Experimental results, given in Fig. 2a and an inset, demonstrate a very fast dynamics of the effect and exhibit a more favorable condition for the enhancement at lower excitation energy, i.e., when the contribution of FC nonlinearity is smaller. The measurements of dc-field induced nonlinear response (described as $\eta\left(E_{\mathrm{dc}}\right) / \eta\left(E_{\mathrm{dc}}=0\right)$ and performed at $\Delta t \cong 30 \mathrm{ps}$ ) indicate that the influence of external bias is much more pronounced at illumination with a larger spatial period of the interference pattern (see Fig. 2b).

We notice that the short electron lifetime in the used samples has not allowed us to reach the nanosecond relaxation regime, favorable for the propagating 
domain grating formation and oscillating nonlinear response, typical of the dcbiased GaAs [3]. Hence, in this paper, the dynamics of the hot-electron nonlinear response is analysed numerically at probe-beam delay times $0 \leq \Delta t \leq 200$ ps.
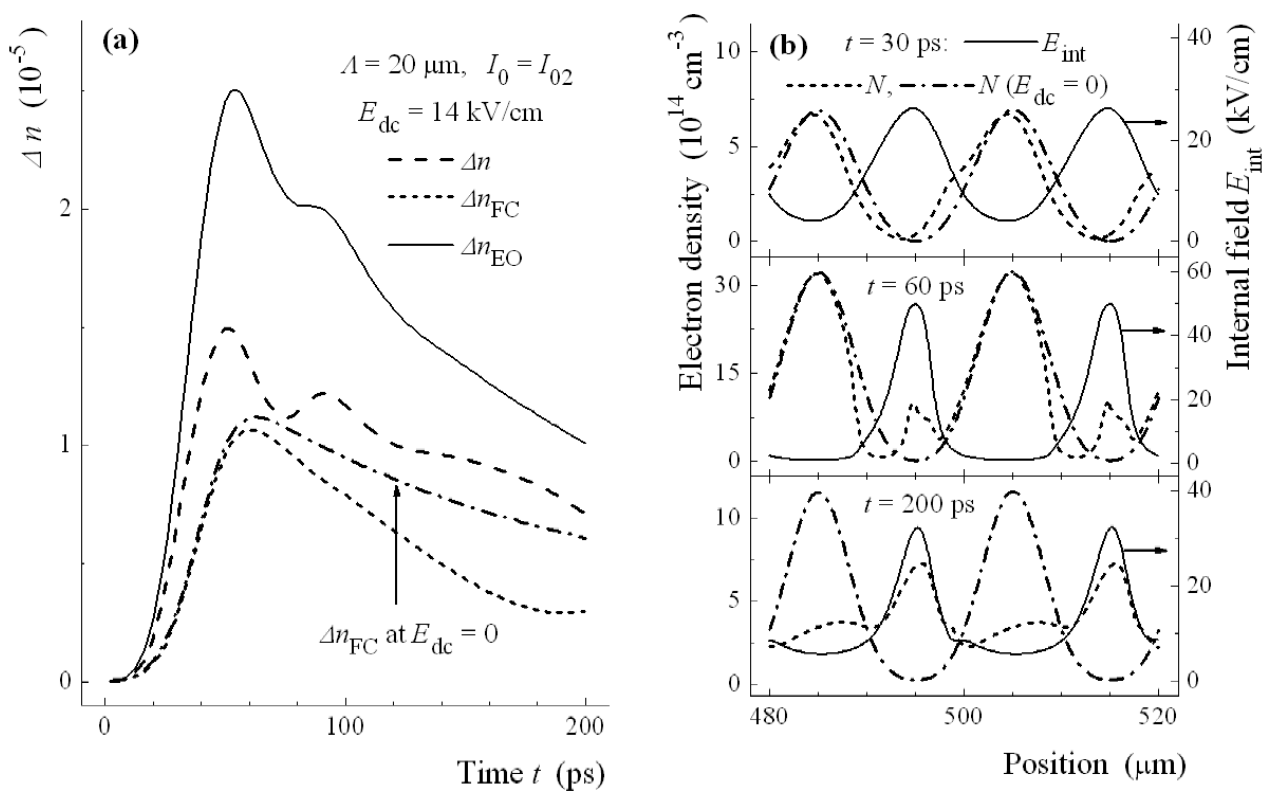

Fig. 3. (a) Time dependence of refraction index modulation $\Delta n$ by FC density $\Delta n_{\mathrm{FC}}$ and EO nonlinearity $\Delta n_{\mathrm{EO}}$, simulated under dc-bias $\left(E_{\mathrm{dc}}=14 \mathrm{kV} / \mathrm{cm}\right)$ by using the same, as in Fig. 1, parameters for the case $I_{0}=I_{02}$. (b) Spatial distribution of electron density and internal electric field (right-hand scale), simulated for $I_{0}=I_{02}$ and $E_{\mathrm{dc}}=$ $14 \mathrm{kV} / \mathrm{cm}$ at three different time-moments. Dash-dot curves - the results in absence of external bias.

In Fig. 3a, we plotted the dynamics of the refractive index modulation $\Delta n(t)$, depending on an instantaneous value of the coexisting optical nonlinearities $\left(\Delta n_{\mathrm{FC}}\right.$ and $\left.\Delta n_{\mathrm{EO}}\right)$, calculated in dc-biased grating at $E_{\mathrm{dc}}=14 \mathrm{kV} / \mathrm{cm}$ and $\Lambda=20 \mu \mathrm{m}$ for excitation intensity of $I_{0}=I_{02}$. Additionally, the plots in Fig. $3 \mathrm{~b}$ demonstrate the instantaneous distribution of electron density and internal field $E_{\text {int }}$ in a central part of $1 \mathrm{~mm}$ long sample, simulated at $E_{\mathrm{dc}}=14 \mathrm{kV} / \mathrm{cm}$ for three different time-moments. The calculation results clearly indicate that the responsible for enhancement optical nonlinearity is due to the quadratic EO effect. One can see (Fig. 3a), that under applied dc-field the FC nonlinearity decreases due to faster carrier grating decay, whereas the EO nonlinearity is effectively enhanced due to the high-field domain formation during the illumination.

A more detailed analysis revealed the light triggered processes of field-shielding and high-field domain grating formation in highly-excited semi-insulating InP. Initially the formation of the EO grating takes place as the exter- 
nal dc-field becomes screened in the peaks of carrier density modulation. Later, with increasing the electron concentration in the regions of NDR, the growth in the SC field is expected due to nonuniform electron heating and the photorefractive Gunn-effect [1]. Similarly, in dc-biased InP the fast effects of hot-electron transport enhance the electric field modulation and develop the high-speed EO nonlinearity which dominates during the action of ps laser pulse.

\section{Acknowledgments}

The work is supported by NATO's Scientific Affairs Division (Science for Peace Programme Project SfP-974476).

\section{References}

[1] L. Subačius, V. Gružinskis, E. Starikov, P. Shiktorov, K. Jarašiūnas, Phys. Rev. $B$ 55, 12844 (1997).

[2] L. Subačius, K. Jarašiūnas, Semicond. Sci. Technol. 16, 864 (2001).

[3] L. Subačius, I. Kašalynas, R. Aleksiejūnas, K. Jarašiūnas, Appl. Phys. Lett. 83, 1557 (2003).

[4] G. Picoli, P. Gravey, C. Ozkul, V. Vieux, J. Appl. Phys. 66, 3798 (1989).

[5] H.J. Eichler, Y. Ding, B. Smandek, Optics Commun. 94, 127 (1992). 